\title{
Radiative-transfer modelling of funnel flows
}

\author{
Tim J. Harries \\ School of Physics, University of Exeter, Stocker Road, Exeter, EX4 4QL, UK \\ email : th@astro.ex.ac.uk
}

\begin{abstract}
Emission line profiles from pre-main-sequence objects accreting via magneticallycontrolled funnel flows encode information on the geometry and kinematics of the material on stellar radius scales. In order to extract this information it is necessary to perform radiativetransfer modelling of the gas to produce synthetic line profiles. In this review I discuss the physics that needs to be included in such models, and the numerical methods and assumptions that are used to render the problem tractable. I review the progress made in the field over the last decade, and summarize the main successes and failures of the modelling work.
\end{abstract}

Keywords. Radiative transfer, line: formation, stars: formation, stars: magnetic fields.

\section{Introduction}

The permitted emission lines from Classical T Tauri stars (CTTS) are thought to arise from the hot funnel flows and winds that occur within a few stellar radii of the stellar photosphere. These lines display a wide variety of morphologies, including double- and single-peaked profiles along with classical and inverse P Cygni absorption dips (see for example Reipurth et al. 1996). The lines often show high levels of variability on a timescale of hours to days, with the former likely to be characterstic of the infall timescale and the latter representing rotational modulation (see contribution by Alencar in these proceedings).

Although the accretion rates are best determined from the blue continuum excess, this is only possible for the highest accretion rates. However the strength and shape of the emission lines are also an accretion rate diagnostic, albeit a more model-dependent one. The broad aim of this field is to find a self-consistent model for the geometry and kinematics of the material as it falls onto, or is ejected from, the protostellar system. In order to construct such a model it is first necessary to have a representation of the magnetosphere, perhaps from a canonical analytical description, or from an MHD model, or even from a magnetic field description derived from observation. This density and velocity structure may then be used in combination with a radiative-transfer code to compute line profiles, and comparison with observations then allows one to refine the magnetospheric description.

We are, frankly, a long way from such a self-consistent model, but the last decade has seen significant advances which mean that the idea of developing such a model is not quite as fanciful as it was 10 years ago. In the following section I detail the physical ingredients that go in to the radiative-transfer model, and then I summarize the main numerical techniques that are used to solve the equation of statistical equilibrium and to compute the profiles. I give a review of the increasingly sophisticated models in subsequent sections, and finish with a critical review of the state-of-the-art. 


\section{The physical model}

The material in the magnetosphere is certainly not distributed in spherical symmetry, and any calculations need to be two, or possibly three, dimensional in nature. The gas is also accelerating, and the velocity pattern is non-monotonic. These properties combine to make the solution of radiative-transfer equations for the magnetospheric a difficult numerical problem, requiring sophisticated modelling codes. Here I detail some of the equations that apply, and the methods and approximations that are used to solve them.

\subsection{Radiative equilibrium}

How hot is the gas in the magnetosphere? How does its temperature vary as a function of height above the stellar surface? These are important questions, as the temperature distribution has a strong effect on the line source function, and therefore the line's shape and morphology. The solution of the radiative equilibrium is, on the face of it, simply a question of comparing the local heating rate with the local cooling rate and iterating on the temperature to balance the two. The problem comes with calculating the rates. The cooling is relatively straightfoward, with processes such as bremstrahlung, collisional recombination, and emission from ionic species all contributing. The heating terms that we can easily identify include adiabatic heating due to the convergence of the flow towards the protostar, and photoionization by UV radiation from the hot spots. However, as with the solar corona, there are almost certainly magnetic heating effects that are very difficult to quantify and are in fact probably dominant.

The only paper I am aware of that explicitly addresses the question of radiativeequilibrium in magnetospheric accretion in CTTS is that of Martin (1996), who considered a simple dipolar field with accretion occuring in free-fall along convergent field lines. He did not include any magnetic heating, and discovered that cooling by Mg II and Ca II was important near the photosphere, and that adiabatic compression was the dominant heating source. However, Hartmann, Hewett \& Calvet (1994) found that line profiles from their radiative-transfer calculations based on the Martin temperature structure do not match observations.

Hartmann, Hewett \& Calvet (1994) proposed temperature structure in which a schematic cooling rate (from Hartmann 1982) is balanced with a volumetric heating rate that scales as $r^{-3}$ (taken to represent magnetic heating from a dipolar field). It was found that this temperature structure resulted in line profiles that better-matched observations, and hence it has been widely-used in subsequent calculations. However it should be noted that its schematic nature is one of the main weaknesses of the current models.

\subsection{Statistical equilibrium}

Formally the equation of statistical equilibrium should be solved simultaneously with that of radiative equilibrium, but as pointed out above the majority of contemporary calculations assume a temperature structure. The equation of statistical equilibrium boils down to

$$
\frac{d N_{i}(\mathbf{r})}{d r}=R_{i}+C_{i}=0
$$

where $N_{i}(\mathbf{r})$ are the level populations (including the continuum) of a particular species, and $R_{i}$ and $C_{i}$ are the radiative and collisional rates into and out of level $i$. The key problem here is that the radiative rates depend on the radiation field, which in turn depends on the level populations: an iterative solution is required. The intensity $I$ of the radiation field may be found by integrating the radiation-transport equation, given by

$$
\mathbf{n} \cdot \nabla I_{\nu}(\mathbf{r}, \mathbf{n})=-k_{\nu}(\mathbf{r}) \phi\left(\nu-\frac{\nu_{0}}{c} \mathbf{n} \cdot \mathbf{v}(\mathbf{r})\right)\left(I_{\nu}-S_{\nu}\right)
$$


where $\mathbf{n}$ is a given direction, $k_{\nu}$ is the line opacity, $S_{\nu}$ is the source function, and $\phi$ is the normalized line profile function.

The integral of the above equation is much simplified if the velocity gradient is large enough, since the line profile term then becomes effectively a delta-function and the integral becomes a summation over the resonance zones along $\mathbf{n}$. This is the Sobolev, or large velocity gradient, approximation which underpins the majority of magnetospheric accretion radiative transfer models in the literature to date. The Sobolev approximation is only valid if the conditions of the gas do not change over the Sobolev length $l_{s}$, given by

$$
l_{s}=\frac{v_{\text {therm }}}{\frac{d v}{d r}}
$$

where $v_{\text {therm }}$ is the thermal line width and $d v / d r$ is the velocity gradient. The approximation is poor at the start of the accretion funnels where the velocity gradient is lowest, and improves near the base of the flows. Furthermore, the Sobolev approximation does not allow for non-thermal line broadening. Finally the Sobolev approximation does not treat density discontinuities properly (such as the surfaces of the accretion streams), since the line optical depth depends solely on the velocity gradient, and it may lead to an overestimate of the line optical depth at such boundaries. The alternative approach is to use comoving frame line transfer, in which Equation 2.2 is integrated formally along many rays in order to adequately estimate the mean intensity $J_{\nu}$. The major disadvantage of this method is that it is much more computationally expensive, although we have developed an algorithm employing co-moving frame transfer (see $\S 7$ ).

\section{Development of the radiative-transfer model}

Hartmann, Hewett \& Calvet (1994) used a simple axisymmetric dipole model for the magnetosphere, with the field lines connecting to the disk at a range of radii around corotation and converging to rings in the northern and southern hemispheres of the protostar. A two-level approximation was used to compute hydrogen line profiles, with the radiation field computed under the Sobolev approximation. It was found that the profiles displayed some of the characteristics of the observations, including asymmetries and inverse P Cygni morphologies.

The same geometrical model was employed by Muzerolle, Calvet \& Hartmann (1998), but the two-level approximation was dropped in favour of a full solution of the statistical equilibrium equation a multi-level hydrogen atom. The line profiles computed using this more complete physical model did not differ significantly from those of Hartmann, Hewett \& Calvet (1994), indicating that the two-level approximation was a good one. It was demonstrated that the profile strengths provided a constraint on the accretion funnel temperatures as a function of mass accretion rate. For a given accretion rate there is an upper-limit to the temperature above which the continuum becomes optically thick and the lines go into absorption, and a lower-limit below which the model line flux does not match observations.

The profiles detailed above were calculated under the Sobolev approximation, which does not allow for broadening of the profiles via line damping. Muzerolle, Calvet \& Hartmann (2001) published models in which the Sobolev approximation was used for the statistical equilibrium equation but the profiles themselves were computed using an exact integration of the transport equation in the co-moving frame - it had previously been shown that the main source of error in the profiles arises from the use of the Sobolev approximation in the computation of the line profiles rather than in the statistical 

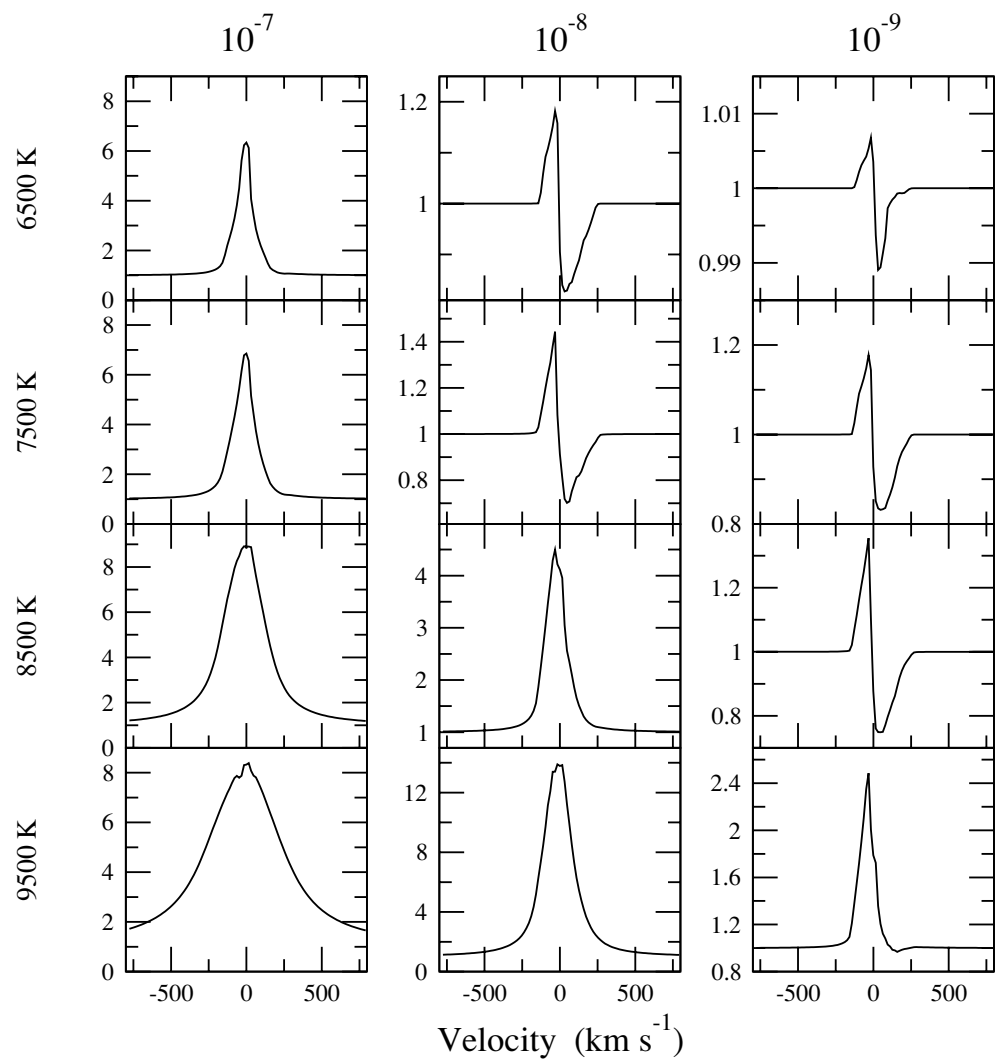

Figure 1. $\mathrm{H} \alpha$ line profiles computed by the TORUs radiative-transfer code and detailed in Kurosawa, Harries \& Symington (2006). The profiles are shown (in velocity space and normalized to the local continuum) as a function of the mass accretion rate and the maximum temperature in the magnetosphere. The models with lower $T_{\max }$ and $\dot{M}$ show classic inverse P Cygni morphology, while the strongest accretors show no evidence for absorption and are broadened significantly by line damping. Similar grids of models have been published by Muzerolle, Calvet \& Hartmann (1998) and Muzerolle, Calvet \& Hartmann (2001).

equilibrium calculation e.g. Bastian et al. (1980). The inclusion of line damping meant that the lower-order Balmer line profiles extended to velocities significantly greater than the terminal velocity of the material, providing much better agreement with observation. This paper was also the first to show a direct comparison (or fit) with the observations of a T Tauri star (specifically BP Tau). We illustrate the kinds of profiles produced using this method in Figure 1.

The developments reviewed here represent a step-change in physical realism over previous models. Nonetheless there are significant problems with these models when they are compared with the observations. Firstly the areal hot spot coverage of the dipolar geometry used in the models is $\sim 8 \%$, which is significantly higher from estimates based on photometric rotational modulation, which indicate about $1 \%$ area coverage. This has implications for both the geometry of the flow and the radiation field from the star. Furthermore the models are two-dimensional and do not produce the variability that seems to be a ubiquitous feature of magnetospheric accretion. Finally since the models only include an inflowing component they cannot reproduce the classical P Cygni profiles that are frequently observed. Recent models have attempted to address some of these shortfalls, and I review these efforts in the following sections. 


\section{Rotational modulation of the emission line profiles}

Rotational modulation of the line profile shapes indicates a departure from axisymmetry in the magnetospheres. Symington, Harries \& Kurosawa (2005) used the canonical description of a dipolar magnetosphere, and broke the symmetry by simply only allowing accretion over a range of azimuthal angles (see Figure 2). This 'accretion curtains' geometry approximated to the situation proposed by observers to explain the rotational variability, and also resembles the geometries predicted by MHD models. From the timeseries synthetic spectra Symington, Harries \& Kurosawa (2005) were able to compute observables, such as temporal variance spectra and cross-correlation matrices, which could be compared with data.

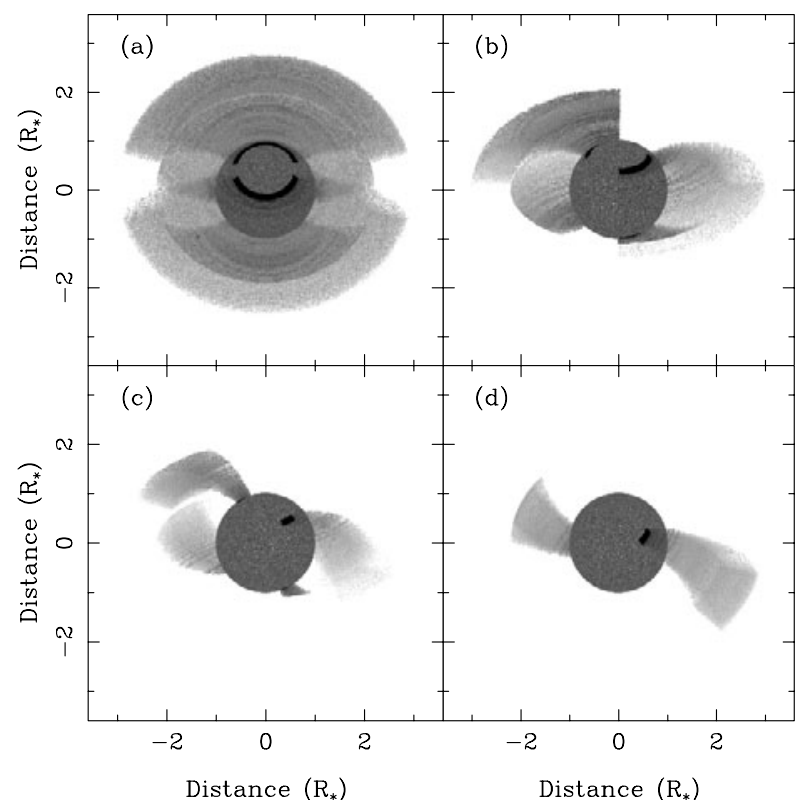

Figure 2. Synthetic $\mathrm{Pa} \beta$ images of the accretion curtains models considered by Symington, Harries \& Kurosawa (2005). The models demonstrate increasing departures from axisymmetry, with curtains of (a) $150^{\circ}$, (b) $90^{\circ}$ (c) $30^{\circ}$ extent. Model (d) has two $30^{\circ}$ curtains in opposite hemispheres, and includes a dipole offet.

It was found that the line variability predicted by most models exceeded the observed variability, excepting those that had high degrees of rotational symmetry. Models with a dipole offset, and narrow accretion curtains (which are similar to the MHD models) displayed line variability far in excess of that observed. It was also observed that although the line variability was generally too high, the continuum variability caused by the hotspots was typically too low. In order to reconcile these differences it is necessary to have a magnetosphere that displays a high degree of axisymmetry at large radii, and then becomes more structured, perhaps by converging into narrow funnels, towards the photosphere.

\section{Models with inflow and outflow}

Magnetospheric accretion is often (always?) accompanied by mass ejection thought to arise via MHD effects e.g. disk winds, stellar winds, jets, X-winds etc. These outflows influence the line profiles principally by adding blue-shifted absorption, but in some 
objects (which show strong double-peaked profiles) there must be significant line emission as well. Distinguishing between the different outflow mechanisms necessitates determining the launching region, and it is possible that a modelling analysis of line profiles will allow this.

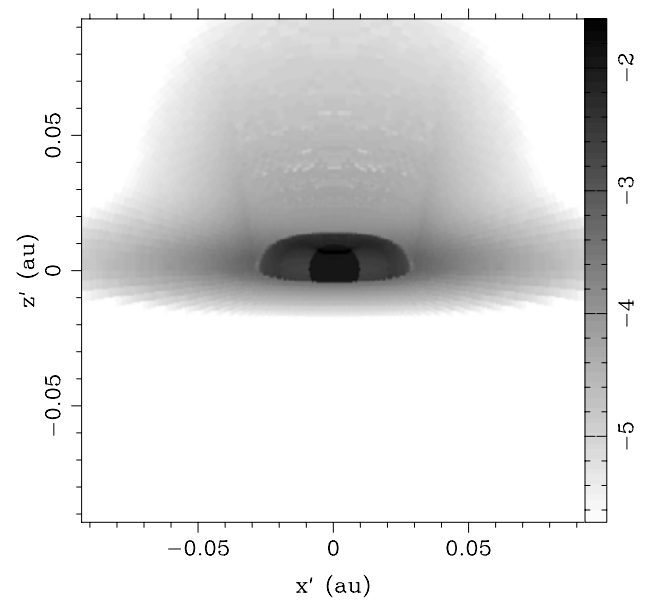

Figure 3. A synthetic $\mathrm{H} \alpha$ image of an accretion plus disk wind hybrid model. The hot rings in the photosphere are clearly visible, along with the strongly emitting magnetosphere and the fainter, but more extended, disk wind emission (Kurosawa, Harries \& Symington 2006).

Recent papers have made progress in this direction. Alencar et al. (2005) studied the CTTS RW Aur, and used a magnetosphere plus wind described by the analytical model of Blandford \& Payne (1982). They performed a full statistial equilibrium calculation under the Sobolev approximation, and found that a magnetospheric-accretion-only model could not reproduce the observed profiles, but that a model in which the outflow emission dominated showed much better agreement. It was found collimated disk winds starting from a small region near the disk inner radius produced the best match, whereas winds with large opening angles were precluded.

A promising direction for research into wind launching is the He I $\lambda 10830$ line, which has a high line opacity and may show classical and inverse P Cygni profiles simulatenously. Kwan, Edwards \& Fischer (2007) published outflow profiles based on a parameterized description of the line emissivity and opacity and using Monte Carlo methods. In summary, these models demonstrate the power of the $\lambda 10830$ line in diagnosing the wind geometry: this work is covered in detail by Edwards' contribution to these proceedings.

Kurosawa, Harries \& Symington (2006) computed grids of $\mathrm{H} \alpha$ profiles based on a canonical magnetosphere and a parameterization of a disk wind (see Figures 3 and 4). It was found that this hybrid model was capable of reproducing the wide variety of observed profile shapes, even with the mass accretion rate to mass-loss rate ratio fixed at 0.1. It is found that the most frequently observed profiles occupied the widest volume of parameter space, whereas the rarest profiles required very specific sets of parameters. We attempted to place the Reipurth et al. (1996) spectral classification system on a more physical basis, by determining what range of parameters are necessary to produce each profile sub-type. We summarize these findings in Figure 5 and Table 1. 

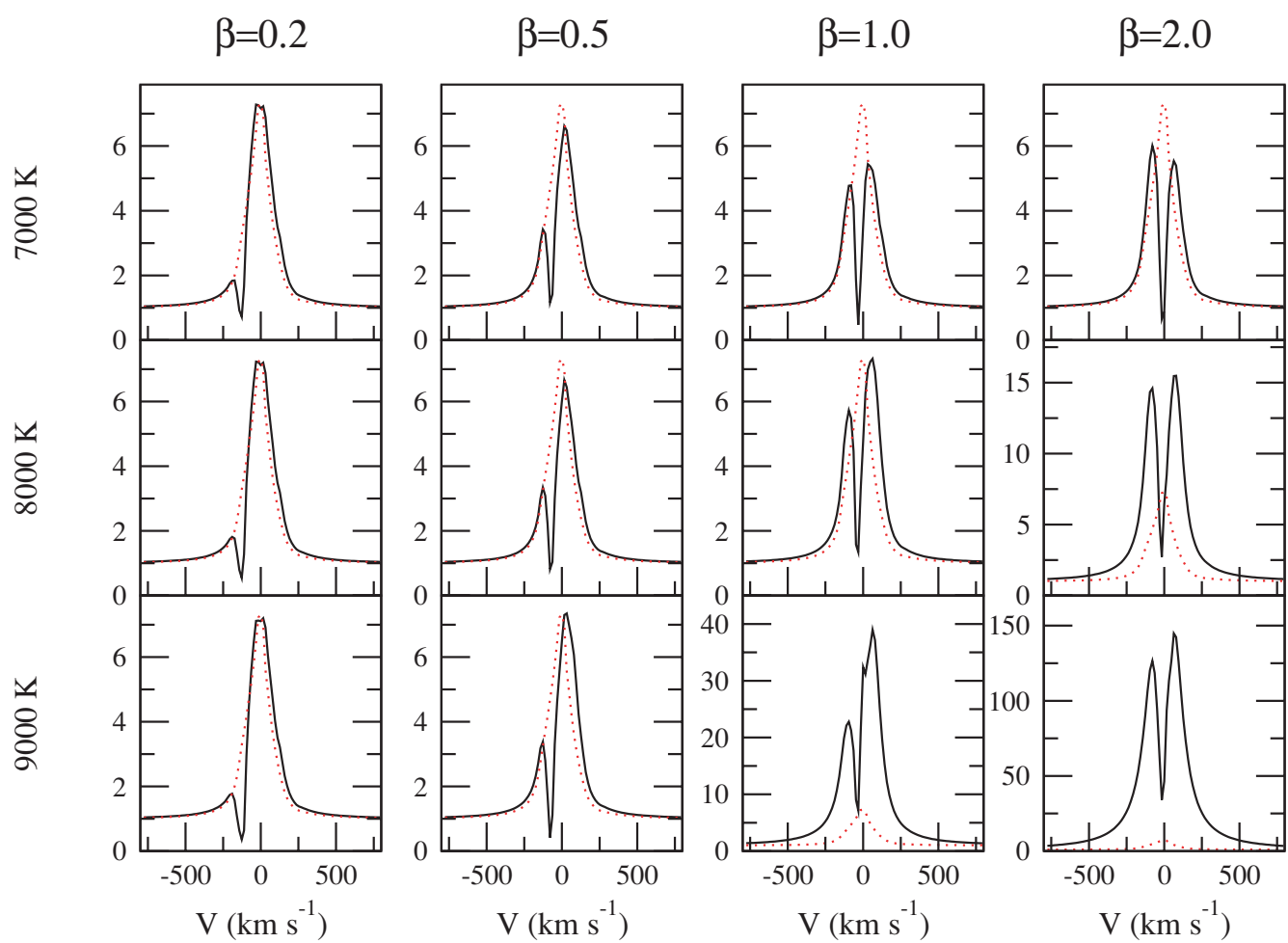

Figure 4. Synthetic $\mathrm{H} \alpha$ line profiles as a function of maximum accretion funnel temperature and wind acceleration parameter, $\beta$. Models with a larger $\beta$ accelerate more slowly, leading to larger densities near the wind-launching region and more line emission from the wind. The models include both the wind and the magnetosphere, and the dotted lines show the equivalent magnetospheric-accretion-only profile. Profiles at the top left of the diagram are dominated by emission from the accretion funnels, those at the bottom right are dominated by the wind.

Table 1. The wind, accretion, and viewing angle requirements for different line profile classes in the Reipurth et al. (1996) classification scheme (from Kurosawa, Harries \& Symington 2006).

\begin{tabular}{ll}
\hline Class & Comment \\
\hline I & Accretion dominated. Wide range of inclinations \\
II-B & Wide range of wind acceleration. Mid-to-high inclination. \\
II-R & Slow wind acceleration rate. High inclination. \\
III-B & Fast wind acceleration. Mid inclination. \\
III-R & Mid wind acceleration. Very high inclination. \\
IV-B & Fast wind acceleration. Mid-to-high inclination. \\
IV-R & Accretion dominated. Low mass-accretion rate. Mid inclination. \\
\hline
\end{tabular}

\section{Models for low- and high-mass protostars}

Attempts have been made to apply the magnetospheric accretion paradigm across the mass spectrum from brown dwarfs (BDs) to Herbig AeBe stars. It is now clear that young BDs share many observational properties with CTTS, including near-to-mid IR excesses indicating the presence of circumstellar disks (e.g. Scholtz et al. 2007) and broad, asymmetric, variable $\mathrm{H} \alpha$ emission that may be a signature of accretion (e.g. Muzerolle et al. 2005; Mohanty et al. 2005). The accretion rates for many of VLM protostars and young BDs seem to be too low to produce a significant blue excess, and the line emission 

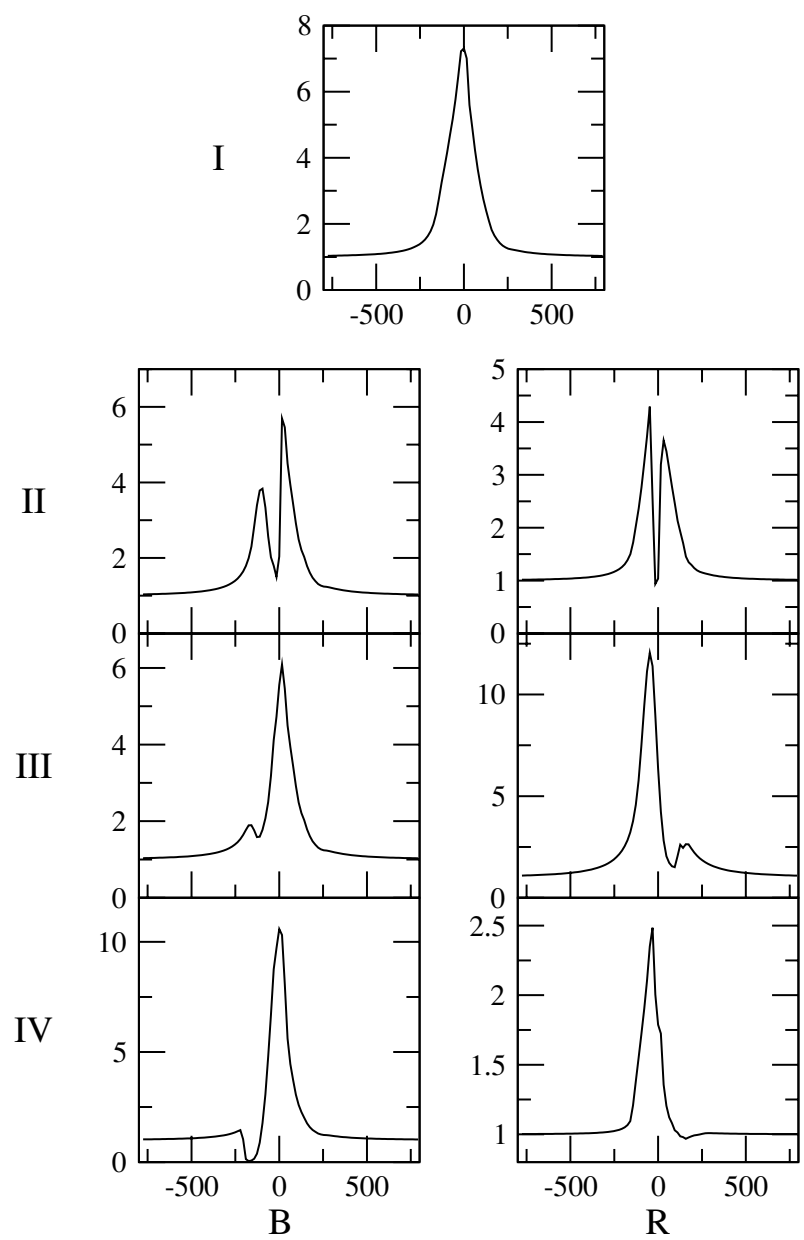

Figure 5. Sample $\mathrm{H} \alpha$ line profiles from the grid computed by Kurosawa, Harries \& Symington (2006), labelled by their Reipurth sub-types. A description of the physical parameters used to compute each of the sub-types is given in Table 1.

is the sole route to measuring the accretion rates. This can be done by extrapolating the line flux/mass accretion rate relationship that is found for higher mass stars (e.g. Natta, Testi \& Randich 2006), or by applying the magnetospheric accretion model e.g. Muzerolle et al. (2005). These studies indicate that the accretion rate drops rapidly with stellar mass (approximately $\dot{M} \propto M^{2}$, see Mohanty's contribution to these proceedings).

It now appears that a simple application of the magnetospheric accretion model to BDs is probably not correct. In order to get sufficient line emission at these low accretion rates Muzerolle et al. (2005) had to increase the temperature of the accretion funnels to $\sim 10 \mathrm{kK}$, at which point the material is completely ionized and optically thin in the line. The justification for this is that the cooling rates, which are density-squared processes, being much less efficient in these rarified streams. However, measurements of the $\mathrm{Pa} \beta$ to $\mathrm{Br} \gamma$ line ratios in BDs (Gatti et al. 2006) suggest that they form in optically thick gas, perhaps in the area around the shocks at the BD surface. Further work is needed here, but the fact that the line fluxes appear to follow (albeit with significant scatter) the same flux/accretion rate relationship as the CTTS suggests that the empirical calibration 
may be sufficient to obtain accretion rates without reference to the radiation-transfer modelling.

Muzerolle et al. (2004) applied the magnetospheric accretion model (including a gas disk) to Herbig AeBe stars, and obtained reasonable matches to the line profiles for UX Ori. However the evidence for magnetic fields in Herbig AeBe stars is somewhat weaker than that for CTTS, and it is yet to be seen whether the magnetospheric accretion model can be successfully and routinely applied at these higher masses.

\section{Magnetic field extrapolation models}

The models reviewed so far are based on canonical descriptions of the magnetosphere, but new methods are emerging which may allow us to generate a more realistic picture of the accretion funnel pattern. The technique of Zeeman Doppler Imaging (ZDI) uses the rotational modulation of the circular polarization signature within photospheric absorption lines to map the strength and polarity of the surface field. The ZDI method has been successfully applied to main sequence objects, and attention is now being focussed on pre-main sequence objects.

The field pattern around the star may be determined, under the assumption of a potential field, by extrapolating field lines from the surface. One may then determine which field lines intersect the disk and have an inward pointing effective gravity-these are the accreting field lines (see Figure 6). The density and velocity along the field lines can be found by assuming a mass-accretion rate and free-fall kinematics e.g. Gregory et al. (2006), Gregory, Wood \& Jardine (2007), and Jardine's and Gregory's contributions to these proceedings.

We have created synthetic spectra based on the extrapolated field geometry, via the following process: First, the stellar surface is divided into a large number of area elements. The density and velocity of the accretion flow immediately above each area element is used to find the kinetic power dumped into that element. This power is assumed to be completely reprocessed at the photosphere and emitted as blackbody radiation, enabling us to assign a hot spot temperature to each element. After solving the statistical equilibrium for 15 levels of hydrogen, line profiles can be computed for any transition and viewing angle using a simple ray-tracing integration of the transfer equation. The line profiles (an example is shown in Figure 6) show some of the characteristic features one would expect of an accreting $\mathrm{T}$ Tauri star, including an asymmetric, broad $\mathrm{H} \alpha$ profile and a strong inverse $\mathrm{P}$ Cygni (IPC) at $\mathrm{H} \beta$. The strength of the IPC profile is due to a combination of the temperature structure we have adopted, and line-damping.

The eventual aim of this research is to perform this analysis on data obtained for CTTS, and to compare the resulting synthetic time-series data with the echelle spectroscopy that was used to create the magnetic field maps. This will provide a strong test of the magnetic field extropolation technique, and may provide a route to a self-consistent model of the magnetosphere.

\section{The successes and failures of the model}

Radiation-transfer models are broadly successful in predicting the gross characteristics of the line profiles in terms of shapes and strengths. Furthermore the models have been applied to individual objects in order to find mass-accretion rates, and the rates determined this way show good agreement with the rates derived using the less model-dependent technique of blue-continuum excess. The few objects that have made a 

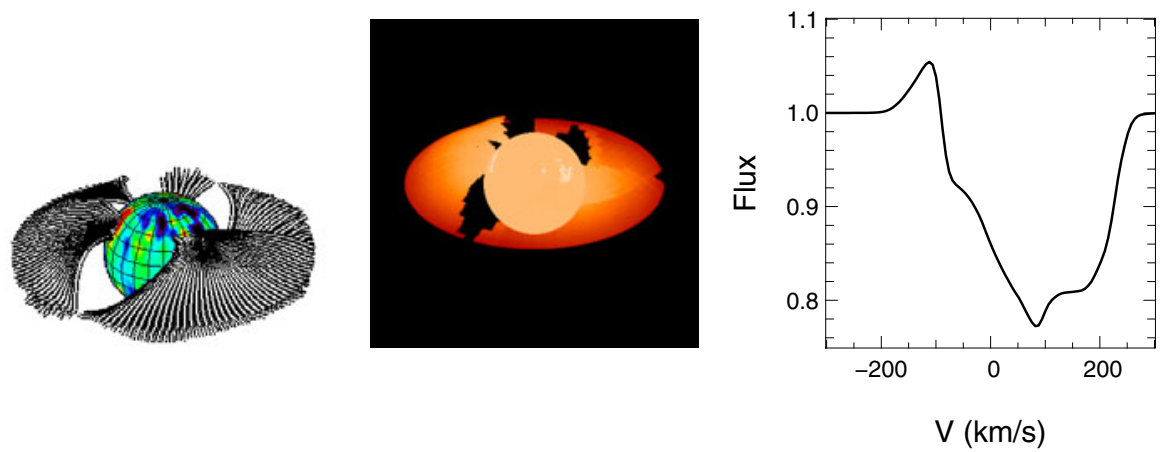

Figure 6. The leftmost figures shows the structure of the accreting magnetic field for a model CTTS with a mass of $0.5 \mathrm{M}_{\odot}$, radius $2 \mathrm{R}_{\odot}$, rotation period $6 \mathrm{~d}$, with an isothermal corona at $20 \mathrm{MK}$ extrapolated from a surface magnetogram of AB Dor. Accretion is assumed to take place over a range of radii within corotation (Gregory, Wood \& Jardine 2007). The figures on the right show an $\mathrm{H} \beta$ intensity image and spectrum respectively, computed in the co-moving frame and based on the geometry of the field extrapolation.

multi-line analysis show that the model works simultaneously over a variety of spectral diagnostics, which is very encouraging.

Progress has also been made in understanding the origin of line profile variability in CTTS, by generating simple accretion curtains models. Rudimentary comparisons have been made between the characteristic variability patterns observed and model grids, and attempts have been made in tailoring such models to individual objects (Kurosawa, Harries \& Symington 2005). It seems that the simple description of the geometry provided by the curtains model is too crude to explain the complex variability seen in the observed profiles. It may be that the magnetosphere is composed of many streams that average to something that approximates to an even azimuthal distribution at large radii, and that then converge as they approach the surface in order to produce the small hotspot areal coverage that is observed.

Several groups are looking at including both inflow and outflow in their radiation transfer models. Although this inevitably leads to yet more free parameters in the models, the inclusion of the outflowing material is vital if the wide-range of line profiles that is observed are to be adequately modelled. Furthermore, analysis of spectral diagnostics that are sensitive to the outflow (e.g. $\lambda 10830$ ) have the potential to strongly constrain the wind-launching mechanism and thus the MHD models. Conversely attempts are being made to produce synthetic profiles based on the MHD modelling (see Romanova's contribution to these proceedings). Once again comparison with the strength, shape, and variability of observed profiles should allow the refinement of the hydrodynamical models.

Despite these successes there are some serious deficiencies in the models that need to be addressed. In particular there needs to be a concerted effort to address the problem of radiative equilibrium in the funnel flows. This is a formidable problem, that has parallels with research into the solar corona. But perhaps if the cooling pathways are properly understood it may be possible to at least constrain the magnetice heating terms? It is also important to try and produce more tailored models for individual targets, using multiple spectral diagnostics to provide the strongest constraints on the array of free parameters in the magnetospheric accretion models. The difficulty here is obtaining observations at sufficient resolution across a sufficiently wide spectral range (preferably the entire visible 
and near-IR regimes) simultaneously, which is necessary to overcome uncertainties due to variability that hamper current studies.

\section{Acknowledgements}

Much of research conducted at Exeter and cited here was performed by Ryuichi Kurosawa and Neil Symington. Scott Gregory and Moira Jardine provided the extrapolated field maps for Section 7, and Christophe Pinte helped with generating the profiles and figures for that section. I thank the SOC for the invitation and the LOC for their hospitality.

\section{References}

Alencar, S.H.P., Basri, G., Hartmann, L., \& Calvet, N. 2005, A\& A 440, 595

Bastian, U., Bertout, C., Stenholm, L., \& Wehrse, R. 1980, A\&6A 86, 105

Blandford, R.D., \& Payne, D.G. 1982, MNRAS 199, 883

Gatti, T., Testi, L., Natta, A., Randich, S., \& Muzerolle, J. 2006, A $\& A$ 460, 547

Gregory, S.G., Wood, K., \& Jardine, M. 2007, astro-ph-07042958

Gregory, S.G., Jardine, M., Collier Cameron, A., \& Donati, J.-F. 2006, MNRAS 373, 827

Hartmann, L. 1982, ApJS 48, 109

Hartmann, L., Hewett, R., \& Calvet, N. 1994, ApJ 426, 669

Kwan, J., Edwards, S., \& Fischer, W. 2007, ApJ 657, 897

Kurosawa, R., Harries, T.J., \& Symington, N.H. 2005, MNRAS 358, 671

Kurosawa, R., Harries, T.J., \& Symington, N.H. 2006, MNRAS 370, 580

Martin, S. 1996, ApJ 470, 537

Mohanty, S., Jayawardhana, R., \& Basri, G., 2005, ApJ 626, 498

Muzerolle, J., Calvet, N., \& Hartmann, L. 1998, ApJ 492, 743

Muzerolle, J., Calvet, N., \& Hartmann, L. 2001, ApJ 550, 944

Muzerolle, J., D’Alessio, P., Calvet, N., \& Hartmann, L. 2004, ApJ 617, 406

Muzerolle, J., Luhman, K.L., Briceño, C., Hartmann, L., \& Calvet, N. 2005, ApJ 625, 906

Natta, A., Testi, L., \& Randich, S. 2006, A\&\&A 452, 245

Reipurth, B., Pedrosa, A., \& Lago, M.T.V.T. 1996, A\&AS 120, 229

Scholz, S., Jayawardhana, R., Wood, K., Meeus, G., Stelzer, B., Walker, C., \& O'Sullivan, M. 2007, ApJ 660, 1517

Symington, N.H., Harries, T.J., \& Kurosawa, R. 2005, MNRAS 356, 1489 

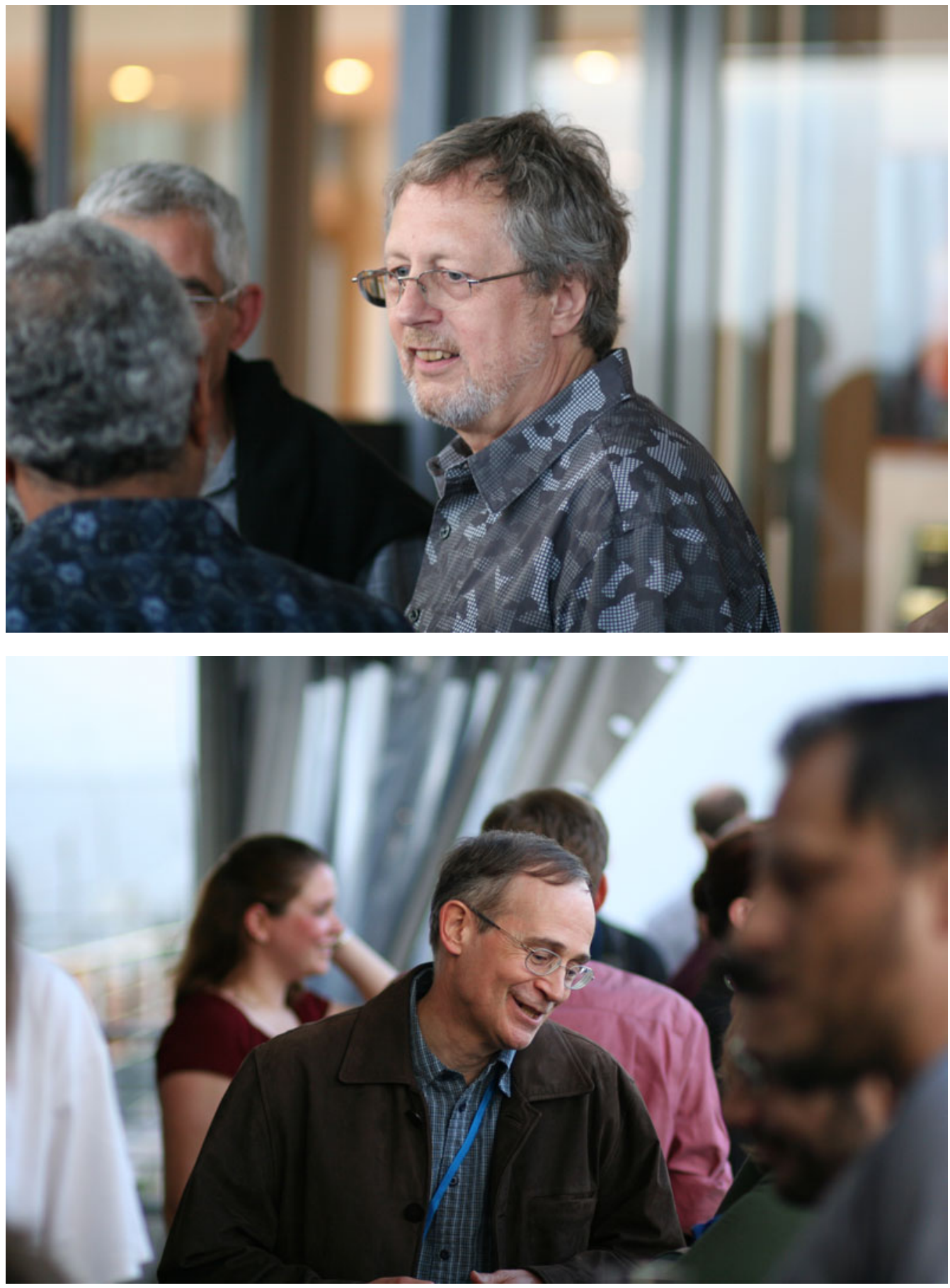